\title{
Performance Analysis of Mm Wave CPW Fed Antenna on Diverse Materials
}

\author{
Iram Nadeem ${ }^{1}$, Jung-jae Lee ${ }^{2}$ and Dong-you Choi ${ }^{1 *}$ \\ ${ }^{1}$ Department of information and communication engineering, Chosun University, \\ South Korea; \\ ${ }^{2}$ Department of computer information science, Songwon University, South Korea; \\ ${ }^{1}$ engineer.iram@gmail.com, ${ }^{2} j j a l e e @$ songwon.ac.kr, ${ }^{1 *}$ dychoi@chosun.ac.kr \\ (Corresponding Author)
}

\begin{abstract}
This paper presents the simulation analysis of CPW (Coplanar waveguide) fed antenna on different substrate for mm wave applications. The antenna was fed by 2 steps modified feed line (rectangular slit and square stub) satisfying the $50 \Omega$ requirement. High Frequency Structure Simulator (HFSS) is employed to analyze the simulation results on the reflection coefficient, VSWR, $H$ - and E-plane radiation pattern and gain of the proposed antenna at various frequencies. The antenna is able to achieve in the range of 65-90 GHz an impedance bandwidth with 9.74 dBi maximum gain value.
\end{abstract}

Keywords: Coplanar waveguide (CPW), millimeter wave (mm-wave), Wave guide, Rectangular slit, square stub, semicircular patch

\section{Introduction}

Recently, the wireless communications system has become integral part of our life and continues to give better quality applications. One of the recent emerging wireless technologies is millimeter wave (mm-wave) technology. It offers various advantages such as large spectral capacity, compact antenna structures and light equipment over the current existing communication systems. The huge band offered by this technology represents great potentials in terms of capacity and flexibility making it particularly attractive for gigabit wireless applications. In other words it has recognized as having ability for broadband ultra-high-speed wireless communication systems such as Giga wireless LAN (Local area network), Gigabit Ethernet networks [1], Wideband radio links for backhaul networking of cellular base stations [2] , automotive radar systems for 77 $\mathrm{GHz}$ [3] and inter-vehicle communication systems. Antennas involved in mm-wave systems mainly concerned in operating bandwidth, gain, radiation efficiency, technological reliability, compatibility with other radio frequency modules and cost. In general there are several techniques for RF transmission lines such as microstrip line, waveguide and coplanar waveguide. However the microstrip antenna [4-5] using a printed board to be patterned by etching is effective in price reduction but it gives significant transmission losses in the millimeter-wave band which in return makes system less efficient. As a solution for this problem directly connect the radiation element and feed line [6] which reduces transmission line losses with high efficiency. Some outdoor applications required bulky components like high gain array or wave guide antenna and connectors. The traditional waveguide are costly with a bulky non-planar hollow and high power capacity which can be synthesized into a planar substrate with metalized slots or even arrays of metallic through vias [7]. Among feeding structures used for wideband antennas coplanar waveguide (CPW) is very attractive due to its characteristics such as low profile, low cost and better isolation from feeding networks. In addition it provides the benefits of good impedance matching, omnidirectional patterns, minimum surface 
wave and easy fabrication. In general, antennas can operate in different frequency bands by adding and / or modifying either to their radiating patch resonator elements of several distinct lengths depending on the lowest frequency band or etching slots in the ground plane. But it makes design too complex which results some drawbacks namely, use of resonator elements generating different resonance modes may increase the size, mutual coupling among signal paths of different resonator parts, complex fabrication process. Therefore it is concluded that most of the techniques used for compact size mm-wave antennas designed have the problems of requiring additional structures, larger size or being too complex to be implemented.

In this paper, we proposed a new compact design approach to achieve simple CPW fed antenna for mm-wave applications. While designing, the selection of substrate material is considered important because it directly affects the electrical properties and radiation efficiency.

\section{Antenna Design}

Figure. 1 shows the geometry of the proposed CPW- fed antenna having size of $18 \times$ $18 \mathrm{~mm}^{2}$. The radiating element is combined with semicircular patch, rectangular patch, square stub and rectangular slit. The Substrate material is Taconic TLY TM with relative permittivity of 2.2 and dielectric loss tangent of 0.0009 respectively.

$$
\mathrm{L}_{\mathrm{Sub}}=\mathrm{W}_{\text {Sub }}=\frac{\mathrm{c}}{2 \mathrm{f}}\left(\frac{\varepsilon_{\mathrm{r}}+1}{2}\right)^{-0.5}
$$

Where $L_{\text {Sub }}$ and $W_{\text {Sub }}$, the length and width of antenna substrate respectively, are equal to the half of the effective wavelength at $f$, the lowest initial operating frequency of antenna. $\mathrm{C}$ is the speed of light in free space and $\varepsilon_{\mathrm{r}}$ is the dielectric constant of substrate. This design has modified feed line structure which satisfies $50 \Omega$ requirements and controllable bandwidth can be achieved. The lower portion of the feed line is a square stub while upper part is a rectangular slit. The feed line is connected to rectangular patch $\mathrm{Wp} \times \mathrm{Lp}$; all are on same side of the substrate. In addition, the two unsymmetrical semicircular patches R1 and R2 in ground plane plays an important role in obtaining wider impedance bandwidth without any cost of size and expense. It offers more electromagnetic wave propagation between radiator and partial ground plane $(a, b, c, d)$ and the careful adjustment of gap between the feed line and radiator has significant impact on impedance bandwidth. Approximate gap values taken are indicated as e and $\mathrm{f}$. The final optimal dimensions of the proposed antenna are as follows: $\mathrm{L}_{\mathrm{Sub}}=18 \mathrm{~mm}, \mathrm{~W}_{\mathrm{Sub}}=$ $18 \mathrm{~mm}$,

$\mathrm{Wp}=6.9 \mathrm{~mm}, \mathrm{Lp}=6 \mathrm{~mm}, \mathrm{~L} 1=4 \mathrm{~mm}, \mathrm{~W} 1=4 \mathrm{~mm}, \mathrm{~L} 2=4.2 \mathrm{~mm}, \mathrm{~W} 2=2.4 \mathrm{~mm}, \mathrm{a}$ $=5 \mathrm{~mm}, \mathrm{~b}=2 \mathrm{~mm}, \mathrm{c}=2 \mathrm{~mm}, \mathrm{~d}=3 \mathrm{~mm}, \mathrm{e}=0.5 \mathrm{~mm}, \mathrm{f}=0.5 \mathrm{~mm}, \mathrm{R} 1=2.6 \mathrm{~mm}, \mathrm{R} 2=$ $2.5 \mathrm{~mm}, \mathrm{~h}=1.62 \mathrm{~mm}$ 


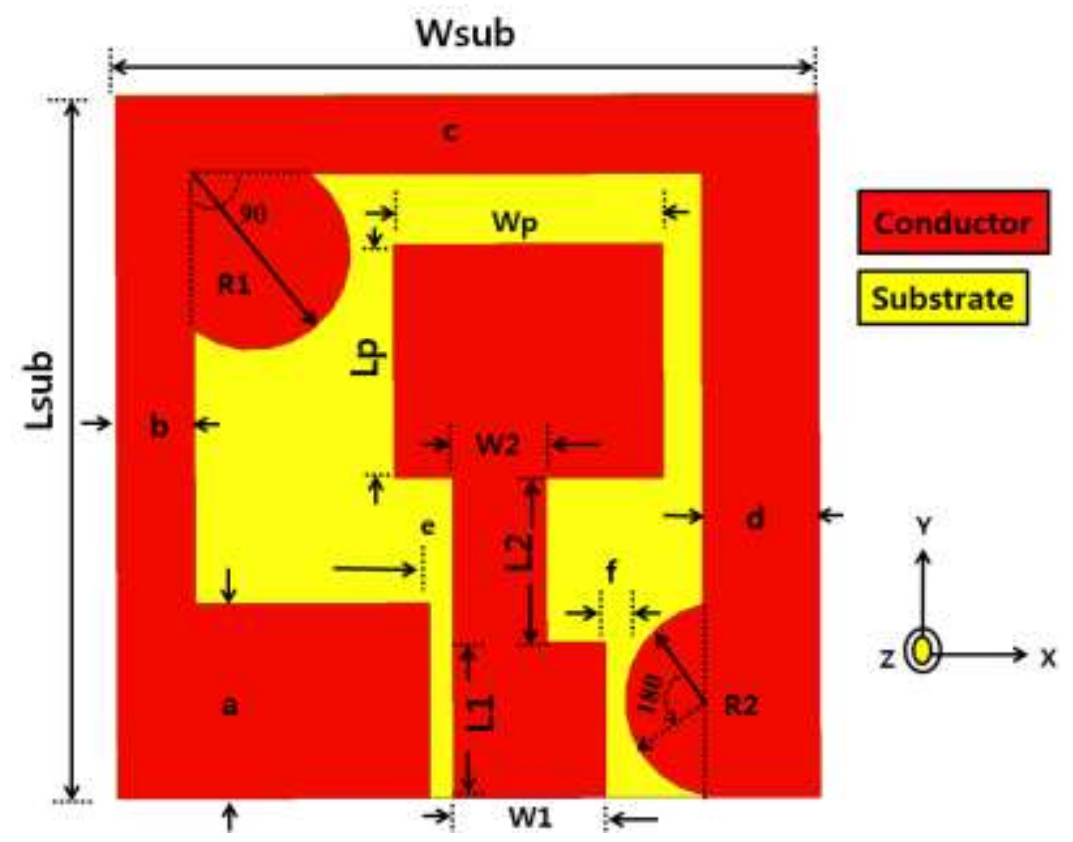

Figure 1. Geometry of the Proposed CPW Fed Antenna

As the selection of the substrate material is very important because it shows the characteristics such as electrical property, environmental resistance and mechanical strength. The electrical property of substrate involves dielectric constant and dielectric loss. Dielectric permittivity is defined as the ratio between the electric field within the material and the corresponding displacement.

$$
\mathrm{D}=\varepsilon \mathrm{E}
$$

The dielectric permittivity and the electric displacement defines that how strongly a material can be electrically polarized under the influence of an electric field. The higher the dielectric constant, the frequency width is narrower. Therefore, dielectric constant of three or less than three is generally suitable for antenna materials [8]. Generally, the dielectric properties of materials are expressed using the relative permittivity $\varepsilon_{\mathrm{r}}$. The relative permittivity defines the dielectric properties of a material relative to that of freespace.

$$
\varepsilon_{\mathrm{r}}=\frac{s}{s_{0}}
$$

When an electromagnetic wave meets interference, some of their energy is reflected and some of their energy is transmitted. For higher frequency EM waves (mm-waves), the portion of reflected energy depends on dielectric properties of the material. For this we focused on four different types of substrate material with less then or higher than three relative permittivity values and evaluated them. Table 1 gives the electrical properties of the materials whereas the resultant effect of reflection coefficients S11 due to these substrate materials while keeping all design parameters same are drawn in Figure.2.

$$
\mathrm{S} 11=\frac{1-\sqrt{\Xi_{\mathrm{r}}}}{1+\sqrt{\varepsilon_{\mathrm{r}}}}
$$


Table 1. Materials and their Corresponding Properties

\begin{tabular}{|l|l|l|}
\hline \multicolumn{1}{|c|}{ Material } & Relative Permittivity & Dielectric Loss Tangent \\
\hline Rogers Ro3210 TM & 10.2 & 0.0035 \\
\hline Rogers RT/duroid 6006 TM & 6.15 & 0.0019 \\
\hline Epoxy FR_4 & 4.4 & 0.02 \\
\hline Taconic TLY TM & 2.2 & 0.0009 \\
\hline
\end{tabular}

It is clear from Figure. 2 that material with high relative permittivity values give lower bandwidth such as Rogers R03210 TM is hardly covering from higher frequency portion S11 (reflection coefficient ) $\leq-10 \mathrm{~dB}$, in other words it represents the worst matching between the radiator and feed line or there is no reflected power. Next, the material is changed to Rogers RT/duroid 6006 TM, whose dielectric loss tangent is half of the previous material and same goes with relative permittivity. Initially, lower frequencies barely touches the good reflection coefficient requirement until it reaches the higher frequencies, max peak of $-21 \mathrm{~dB}$ is observed at nearly $87 \mathrm{GHz}$. This shows that there is only higher order mood resolution on $87 \mathrm{GHz}$ and all power is reflected with low power loss. Furthermore, the material is replaced with Epoxy FR_4, a clear change is observed for whole bandwidth with maximum reflection coefficient value of $-13 \mathrm{~dB}$. The higher the absolute value of the reflection coefficient the performance is considered better because of low power losses.

Lastly, Taconic TLY TM having lowest relative permittivity of 2.2 is used which is the material used for our proposed design. Figure. 2 supports the point that the dielectric loss affects the loss in the feed line, radiator and if the losses become high, radiation efficiency is declined. Therefore dielectric loss and relative permittivity is a critical indicator in designing antenna for $(\mathrm{mm})$ millimeter-wave applications. Table 2 gives the operating frequency bandwidths along with simulated gain values. So the difference in gain values is very prominent as it is the combination of the directivity and efficiency.

\section{Simulation Analysis and Results}

The parameters are optimized using the simulation software HFSSv12 (High Frequency Simulator Software). The simulation reflection coefficient (S11) and VSWR of the optimal antenna are shown in Figure. 3 (a) and (b) respectively.

The proposed antenna frequency ranges from $65 \mathrm{GHz}$ to $90 \mathrm{GHz}$ which gives the total bandwidth of $25 \mathrm{GHz}$. According to antenna theory [9] (reflection coefficient) S11 should be $\leq-10 \mathrm{~dB}$ and VSWR must be $\leq 2$ for good impedance matching between the feed line and radiator patch in certain frequency bands as well as to the presence to rectangular ground plane.

Table 2. Relative Permittivity Effect on Bandwidth and Peak Realized Gain

\begin{tabular}{|c|c|l|r|r|r|}
\hline $\begin{array}{l}\text { Substrate } \\
\text { thickness } \\
(\mathbf{m m})\end{array}$ & \multirow{2}{*}{$\begin{array}{l}\text { Relative } \\
\text { permittivity }\end{array}$} & $\begin{array}{l}\text { Operating } \\
\text { frequency } \\
\text { band }(\mathbf{G H z})\end{array}$ & \multicolumn{3}{|c|}{ Peak realized Gain (dBi) } \\
\cline { 3 - 6 } & & $\mathbf{7 0 ~ G H z}$ & $\mathbf{8 0 ~ G H z}$ & \multicolumn{1}{|c|}{$\mathbf{9 0 G H z}$} \\
\hline 1.62 & 10.2 & \multicolumn{1}{|c|}{$82-85$} & 4.76 & 6.36 & 6.6 \\
\hline 1.62 & 6.15 & $\begin{array}{l}65-71,74-78, \\
82-86\end{array}$ & 5.77 & 5.87 & 6.9 \\
\hline 1.62 & 4.3 & $\begin{array}{l}65-79,81-86, \\
89-90\end{array}$ & 6.08 & 6.10 & 7.04 \\
\hline 1.62 & 2.2 & $65-90$ & 8.12 & 8.14 & 9.74 \\
\hline
\end{tabular}




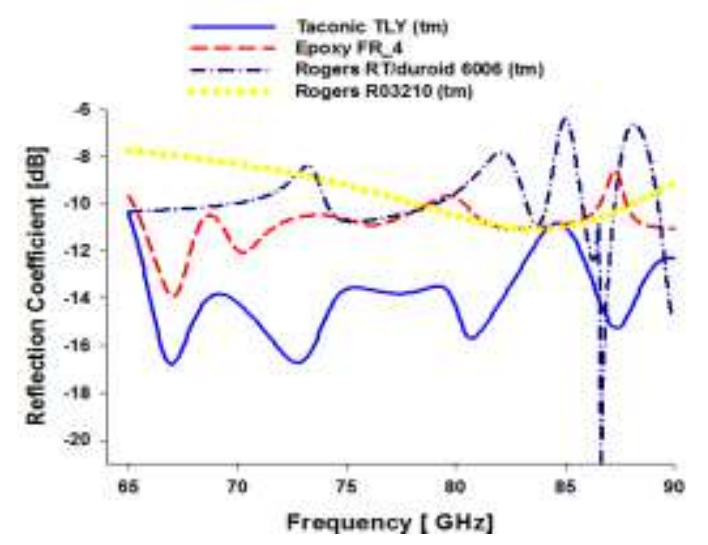

Figure 2. Reflection Coefficient Comparison among Various Materials

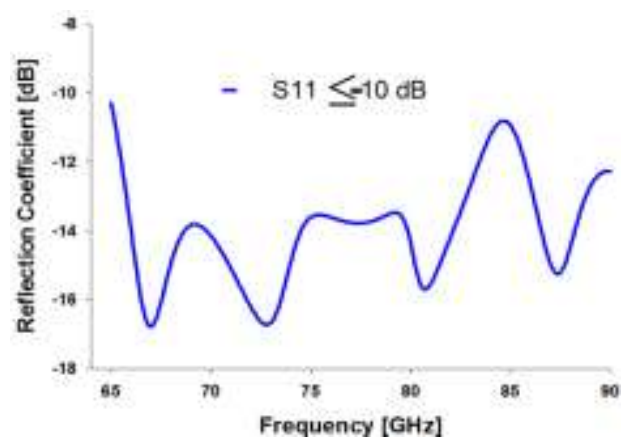

(a)

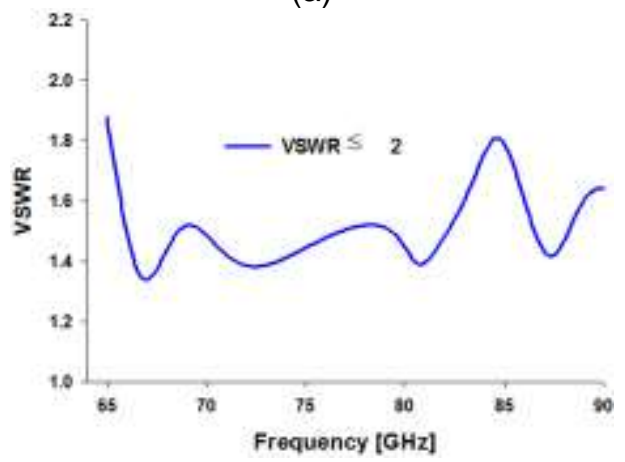

(b)

Figure 3. (a) Simulated Reflection Coefficient, (b) VSWR of the Proposed CPW Fed Antenna

The simulated H-plane (yoz) and E-plane (xoz) radiation pattern at $70 \mathrm{GHz}, 80 \mathrm{GHz}$ and $90 \mathrm{GHz}$ are plotted in Figure.4 (a) and (b), respectively. In $\mathrm{H}$ plane, the desirable antenna shows a nearly directional radiation pattern. Where as in E-plane a dipole like radiation pattern is observed. With the increase in frequency the radiation pattern becomes more distorted. The simulated peak realized gain value varies from $8.12 \mathrm{dBi}$ to $9.74 \mathrm{dBi}$ which predicts that proposed antenna exhibits very high gain as shown in Figure. 5. 


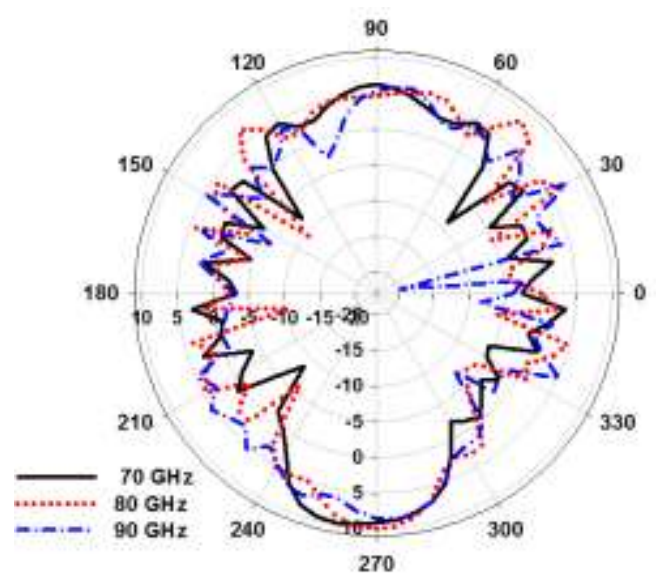

(a)

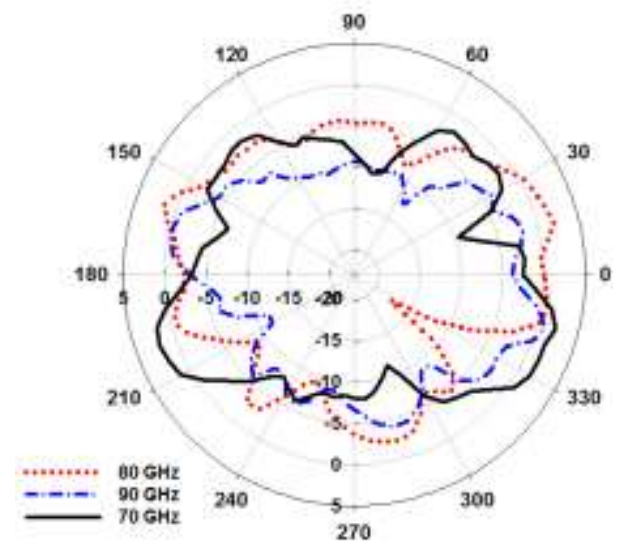

(b)

Figure 4. Simulated Radiation Pattern of the Proposed CPW Fed Antenna (a) H-Plane, (b) E-plane

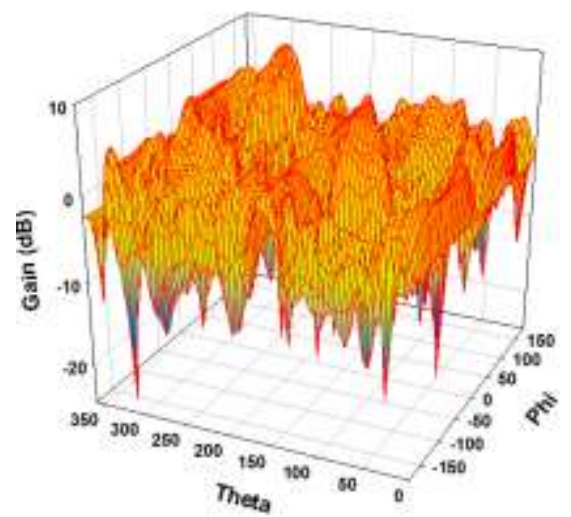

$70 \mathrm{GHz}=8.12 \mathrm{dBi}$ 


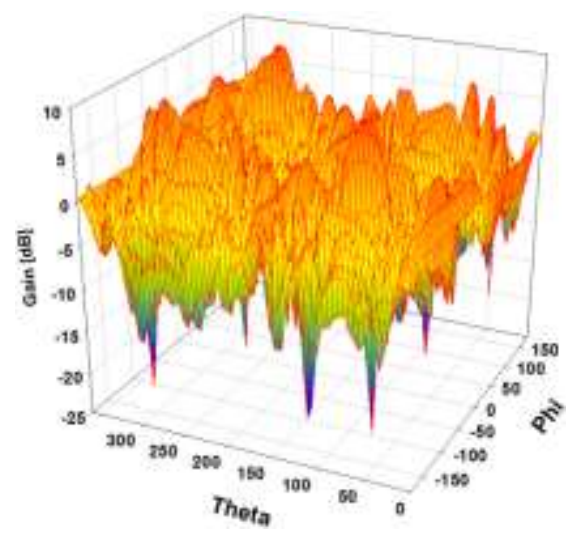

$80 \mathrm{GHz}=8.14 \mathrm{dBi}$

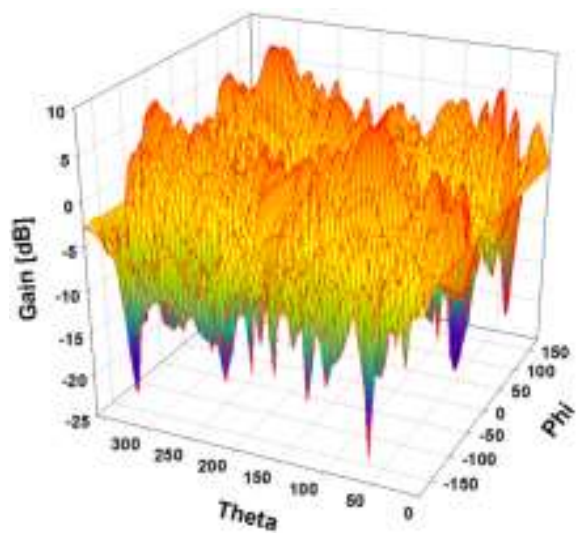

$90 \mathrm{GHz}=9.74 \mathrm{dBi}$

Figure 5. Simulated 3 D Peak Realized Gain (dB) of the Proposed CPW Fed Antenna

\section{Conclusion}

In this paper, simulation analysis of a unique CPW fed antenna with an interesting structure has been proposed which contributes to the investigation of mm-wave applications. The effect of electrical properties on substrate material is shown in it. The proposed antenna covers bandwidth range from $65 \mathrm{GHz}$ to $90 \mathrm{GHz}$. The $\mathrm{H}$-plane and Eplane radiation pattern shows the proposed design has directional effects and exhibits very high peak realized gain ranging from 8.13 to $9.74 \mathrm{dBi}$. Hence, the propose design is simple in nature; acquire smaller surface area and good gain values over the entire operating bandwidth.

\section{Acknowledgement}

This research was supported by the Basic Science Research Program through the National Research Foundation of Korea (NRF) funded by the Ministry of Education (2016R1D1A1B03931806).

\section{References}

[1] A. Azzemi, D. Isa and A. M. mohammadi, " Broadband Transition from Microstrip Line to Waveguide Using a Radial Probe and Extended GND Planes for Millimeter-Wave Applications", Progress In Electromagnetics Research Letters, vol. 60, (2016), pp. 95-100.

[2] Z. A. Saad Z, "E-band microwave radios for mobile backhaul", International Journal of Wireless and Microwave Technologies (IJWMT), vol. 5, no.4, (2015), pp. 37-46. 
[3] I. Gresham, N. Jain, T. Budka, A. Alexanian, N. Kinayman, B. Ziegner, S. Brown and P. Staecker, "A compact manufacturable 76-77-GHz radar module for commercial ACC applications", IEEE Transactions on Microwave Theory and Techniques, vol. 49 , no.1, (2001), pp.44-58.

[4] I. Nadeem, S. Shrestha, S. W. Kim, S J. Han and Y. D. Choi, "Design of slotted UWB Antenna with modified Ground Plane for Bio Medical Applications", In proceedings of the international conference on Electronics, Information and Communication, Phuket, Thailand, (2017), pp. 621-624.

[5] I. Nadeem and Y J. Kim, "Design of Ultra-Wide Band Antenna with Compact MCR Structure for BW Enhancement", The Journal of the Korean Institute of Communication Sciences, vol. 42, no. 4, (2017), pp.798-805.

[6] I. Hideo, T. Watanabe, S. A. T. O. Kazuo, and K. Nishikawa, "Millimeter-wave microstrip line to waveguide transition fabricated on a single layer dielectric substrate", IEICE Transactions on Communications, vol. 85, no. 6, (2002), pp. 1169-1177.

[7] C. Hailin, F. Jiang, J. Liu, W. Cai, M. Tang, X. Tan and S. Yang, "A CSRR-fed SIW cavity-backed fractal patch antenna for wireless energy harvesting and communication", Sensors, vol. 15, no. 9, (2015), pp. 21196-21203.

[8] Information on http://www.fujitsu-ten.com/business/technicaljournal/pdf/36-3.pdf [Online 15-03-2017]

[9] S. L. Warren and G. A. Thiele, “Antenna theory and design”, John Wiley \& Sons, (2012).

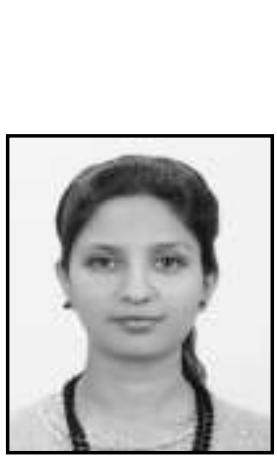

\section{Authors}

Iram Nadeem, she received her M.S. in Telecommunication Engineering (Major: Optical Fiber Communication) and her B.S. in Electrical Engineering from the University of Engineering and Technology (UET) Taxila, Pakistan in 2014 and 2010. She is currently pursuing her Masters in Information and Communication Engineering from Chosun University. She had worked as Lecturer and Lab Engineer in different engineering institutes of Pakistan. Her research interests include optical fiber communication, microwave and satellite communication, antenna design, and wave propagation.

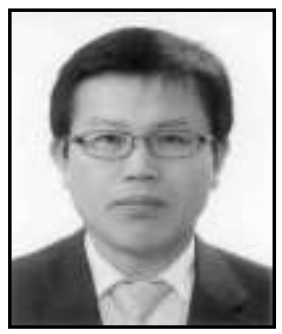

Jung-Jae Lee, he received his B.S., M.S., degrees from the Department of Computer Engineering, Chosun University, Gwangju, Korea, in 1986, 1989. He received his Ph.D. degrees from the Department of Computer \& statistical, Chosun University, Gwangju, Korea, in 1997. Since 1997, he has been a researcher and teaching as a professor, Songwon University, Gwangju, Korea. His research interests include medical antenna design, medical WBAN. He is a member of the KIPS, KIECS.

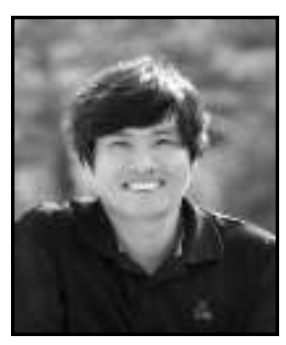

Dong-You Choi, he received his B.S., M.S., and Ph.D. degrees from the Department of Electronic Engineering, Chosun University, Gwangju, Korea, in 1999, 2001, and 2004, respectively. Since 2006, he has been a researcher and teaching as a professor. His research interests include rain attenuation, antenna design, wave propagation, and microwave and satellite communication. He is a member of the IEEE, IEICE, JCN, KEES, IEEK, KICS, and ASK. 